\title{
Direct sludge freezing using dry ice
}

\author{
D.S. Jean ${ }^{a}$, D.J. Lee ${ }^{a, *}$, C.Y. Chang ${ }^{a, b}$ \\ ${ }^{a}$ Department of Chemical Engineering, National Taiwan University, Taipei 10617, Taiwan \\ ${ }^{\mathrm{b}}$ Graduate Institute of Environmental Engineering, National Taiwan University, Taipei 10617, Taiwan
}

\begin{abstract}
Dry ice was used as the heat sink in sludge freeze/thaw treatment. Four distinct sludges were frozen at four thermal contact configurations with dry ice. Experimental results indicate that dry ice freezing can sufficiently condition the sludges and is an economically feasible alternative for sludge conditioning. With a properly designed thermal contactor, the freezing rate can range from 0.48-0.64 m daily. (c) 2001 Elsevier Science Ireland Ltd. All rights reserved.
\end{abstract}

Keywords: Dry ice; Freezing; Sludge; Dewaterability

\section{Introduction}

Freeze/thaw treatment, a highly effective sludge dewatering technique, has received considerable attention (Vesilind and Martel, 1990). Freeze/thaw treatment can significantly improve certain sludge dewatering characteristics, transform the floc structure into a more compact form (e.g. Vesilind et al. (1991)), and reduce the sludge bound water content (Lee and Hsu, 1994). In addition, the underlying mechanisms of the freeze/thaw treatment have received increasing interest (Hung et al., 1996, 1997; Chu et al., 1997; Jean and Lee, 1999; Parker and Collins, 1999). Although highly promising in terms of enhancing dewaterability and for reducing residual moisture content of sludge, the freeze/thaw technique is limited, mainly owing to the high operating cost of the mechanical freezing technique. Martel et al. (1998) reviewed the recent trends

\footnotetext{
* Corresponding author. Tel.: +886-2-2362-5632; fax: $+886-2-2362-3040$.

E-mail address: djlee@ccms.ntu.edu.tw (D.J. Lee).
}

in freezing beds. In tropical or sub-tropical regions, natural freezing of sludge is infeasible.

As the greenhouse gas contributes most to global warming, $\mathrm{CO}_{2}$ has captured global attention since the Kyoto Conference, Framework Convention on Climate Change in terms of the ongoing efforts to contain and effectively manage it. With the advent of cost-effective technological alternatives, $\mathrm{CO}_{2}$ can be efficiently removed from the flue gas of power stations (Hanisch, 1999). Chinese Petroleum Corporation (CPC, Taiwan) imports liquidified natural gas (LNG) using a receiving unit located on the seashore of southern Taiwan. The receiving unit evaporates LNG into a gaseous state and, simultaneously, extracts a vast amount of energy from the surrounding environment. Such a 'cold-energy' process can be used to recover $\mathrm{CO}_{2}$ in the flue gas stream of combustion power plants and convert it to liquidified or solidified forms. At atmospheric pressure, only the solidified form, i.e. dry ice, can stably exist at temperatures less than $-78^{\circ} \mathrm{C}$. Meanwhile, the CPC has its own sludge disposal problem and must meet the standards set in the new environmental regulations activated in 1999. This work examines the feasi- 
bility of using dry ice to condition their sludge. Freezing tests for two other sludges are compared with those of CPC's sludges.

\section{Experimental}

\subsection{The sludge samples}

Four sludge samples were tested: waste activated sludge; oily sludge; sewage sludge; and $\mathrm{Fe}(\mathrm{OH})_{3}$ sludge. The waste activated sludge sample and the oily sludge were taken from the wastewater treatment plant at Chinese Petroleum Corporation, Taoyuan, Taiwan. Testing was commenced within $2 \mathrm{~h}$ after sampling. The chemical oxygen demand (COD), suspended solids (SS) and turbidity data for the supernatant of waste activated sludge were determined using standard methods of the environmental protection administration (EPA) of Taiwan. The results for activated sludge were: 5.6$\mathrm{mg} / \mathrm{l}$ (COD); 7.1-mg/l (SS); and 1.39-NTU (turbidity). The weight percentage of dry mass in the original sludge was $1.51 \%$. The particle size distribution (PSD) was determined using a particle size analyzer (Coulter LS230). The floc size of the original sludge was $70 \mu \mathrm{m}$.

For the oily sludge, the weight fraction of the oily phase was $22.9 \%$. The densities of the supernatant and of the solid were 960 and $1813 \mathrm{~kg} / \mathrm{m}^{3}$, respectively. The mean particle size of the original sludge was 10 $\mu \mathrm{m}$.

A sewage sludge sample was taken from the reflux stream of recycled sludge at the Min-Shen Sewage Treatment Plant, Taipei. The solid weight percent was $2.47 \%$. The COD, SS and turbidity data for the supernatant were: $50.0-\mathrm{mg} / \mathrm{l}$ (COD); $13.8-\mathrm{mg} / \mathrm{l}$ (SS); and 14.6-NTU (turbidity), respectively. The mean particle size of the original sludge was approximately $31 \mu \mathrm{m}$.

Ferric hydroxide slurry was prepared by mixing $54 \mathrm{~g}$ of $\mathrm{FeCl}_{3}$ and $24 \mathrm{~g}$ of $\mathrm{NaOH}$ in a $387-\mathrm{g}$ deionized water bath to produce a precipitate of $21.4 \mathrm{~g}$. The precipitate was flushed several times to remove the by-product sodium chloride. Finally, deionized water was added to the sludge to make a final weight percent of $2.67 \%$. The particle size distribution was monodispersed with a mean diameter of approximately $61.7 \mu \mathrm{m}$. The true solid density was measured as $3710 \mathrm{~kg} / \mathrm{m}^{3}$.

\subsection{Freezing test}

Freezing tests were conducted in a cylindrical freezing chamber having a height of $11.5 \mathrm{~cm}$ and inner diameter of $8.25 \mathrm{~cm}$. Dry ice was cut into cubes of approximately $1 \mathrm{~cm}$. Four configurations were adopted that allowed thermal contact between the original sludge and the dry ice. Configurations 1-3 permitted direct contact between the sludge and dry ice, while configuration 4 permitted indirect contact.

In configuration $1,75 \mathrm{~g}$ of dry ice was placed on the bottom of the freezing chamber. The original sludge $(200 \mathrm{~g})$ at room temperature was then poured on the dry ice. The sludge layer thickness was approximately 3 $\mathrm{cm}$. The sludge layer was vigorously agitated by the sublimated $\mathrm{CO}_{2}$ gas until the sludge was completely frozen. The typical contact time for complete sludge freezing in configuration 1 was $6 \mathrm{~h}$.

The freeze/thaw literature generally recommends using thin layers of sludge for freezing (Martel et al., 1998). In configuration $2,75 \mathrm{~g}$ of the dry ice was initially placed on the bottom of the freezing chamber. The original sludge was then placed on the dry ice at a thickness of $1 \mathrm{~cm}$. After the sludge was completely frozen, another $1-\mathrm{cm}$ of the original sludge was placed on top of the frozen sludge. This process was repeated three times until a total sludge amount of $200 \mathrm{~g}$ was reached. The typical contact time for configuration 2 was $2 \mathrm{~h}$, i.e. shorter than that for configuration 1 .

Configuration 3 employed three layers of original sludge and four layers of dry ice. The first layer of dry ice $(18.75 \mathrm{~g})$ was placed on the bottom of the freezing chamber, and a $1-\mathrm{cm}$ sludge layer was poured onto it. A layer of dry ice of $18.75 \mathrm{~g}$ was immediately placed on the first sludge layer, and then was covered by another layer of sludge. This procedure was repeated. Finally, the three original sludge layers were cooled from both sides by four dry ice layers. The total feeding time was less than $1 \mathrm{~min}$. The effects of feeding time were assumed to be negligible. The typical contact time for complete freezing of the sludge was substantially shorter than for configurations 1 and 2, only ranging from 15 to $20 \mathrm{~min}$.

For the oily sludge tests, some samples were placed in glass tubes of $1-\mathrm{cm}$ diameter and $15-\mathrm{cm}$ length immersed in a pool of dry ice. This arrangement is referred to herein as configuration 4 , and allowed no direct thermal contact between the oily sludge and the dry ice. The typical contact time was approximately $2 \mathrm{~h}$.

For comparison, some samples were placed in a cool room temperature of $-17^{\circ} \mathrm{C}$ for $24 \mathrm{~h}$. These sludge samples were termed air-cooled sludges. In addition, some samples were suddenly immersed into a saturated liquid $\mathrm{N}_{2}$ pool $\left(-198^{\circ} \mathrm{C}\right)$ for $3-5 \mathrm{~min}$, which imitated an ultra-fast freezing. Complete freezing of sludge was achieved within a few seconds.

\subsection{Sludge characteristics}

All frozen samples were thawed at room temperature for $24 \mathrm{~h}$. A capillary suction apparatus described by Lee and Hsu $(1992,1993)$ was employed to estimate the sludge filterability. The inner cylinder radius was $0.535 \mathrm{~cm}$, while the time required for the filtrate to 
Table 1

Experimental results: activated sludge

\begin{tabular}{lcccc}
\hline & ZSV $(\mu \mathrm{m} / \mathrm{s})$ & $\mathrm{CST}(\mathrm{s})$ & $h_{\mathrm{f}} / h_{\mathrm{I}}$ & Solid content $(\% \mathrm{w} / \mathrm{w})$ \\
\hline Original & - & 43.7 & 0.97 & 6.25 \\
Air-cooled & 360 & 31.5 & 0.07 & 11.0 \\
Configuration 1 & 76.1 & 33.9 & 0.35 & 15.7 \\
Configuration 2 & 88.0 & 36.0 & 0.55 & 13.6 \\
Configuration 3 & 150 & 30.5 & 0.25 & 18.3 \\
$\mathrm{~N}_{2}$ & 50 & 37.6 & 0.30 & 8.65 \\
\hline
\end{tabular}

penetrate the filter paper from 1.5 to $3.0 \mathrm{~cm}$ was defined as the capillary suction time (CST). Whatman No. 17 filter paper was used.

After the CST test, a cake formed on the filter paper. The moisture remaining in the cake formed on the filter paper after the CST test was measured by weighing and drying the cake. This residual moisture reflects the amount of bound water in the sludge.

Hindered settling tests were performed in settling tubes of diameter $1 \mathrm{~cm}$ and height $15 \mathrm{~cm}$ after the sample thawed. Strong wall effects were found in these tests. However, the wall effects were expected to largely cancel each other out between tests since only the relative differences between sludge samples were of interest. The ZSV (zone settling velocity) was obtained by linear regression of the interface height vs. time data for the constant-rate period with a regression coefficient exceeding 0.98. Additional experimental details are provided by Chen et al. (1996a,b). The equilibrium sediment height after 24-h settling was recorded as $h_{\mathrm{f}}$. The ratio of $h_{\mathrm{f}} / h_{\mathrm{i}}$, where $h_{\mathrm{i}}$ represents the initial height, provides an index for the bound moisture and structure of the sludge flocs.

Three independent tests were conducted under each condition to check the data reproducibility. The variance in measurements was generally small and only the mean values are reported herein.

\section{Results}

Tables 1-4 summarize the experimental results. ZSV, CST, final sediment height, and solid content in filter cake represent the settleability, filterability, compactness of sludge flocs and the binding strength between moisture and the solid surface, respectively.

\subsection{Activated sludge}

Consider the activated sludge test. Table 1 reveals that the original sludge could barely settle (nearly zero ZSV and close-to-unity $h_{\mathrm{f}} / h_{\mathrm{i}}$ ), but was relatively easy to filter (not a very high CST). In addition, the residual moisture content in the filter cake was extremely high, exceeding $93 \% \mathrm{w} / \mathrm{w}$.

All freezing tests yielded an improved dewaterability. Among all tests, the air-cooled sludge exhibited the best settleability $(\mathrm{ZSV}=360 \mu \mathrm{m} / \mathrm{s})$ and filterability $(\mathrm{CST}=31.5 \mathrm{~s})$. The corresponding final sediment height was also the lowest one $\left(h_{\mathrm{f}} / h_{\mathrm{i}}=0.07\right)$. Such an observation corresponds to our previous work in which a slow freezing process markedly enhanced sludge dewaterability (Hung et al., 1996).

All the configurations for the dry ice-freezing tests significantly enhanced dewaterability. The ZSV ranged from $76-150 \mu \mathrm{m} / \mathrm{s}$, CST was less than $36 \mathrm{~s}, h_{\mathrm{f}} / h_{\mathrm{i}}$ was $0.25-0.55$ and most significantly, the solid contents all exceeded $13.6 \% \mathrm{w} / \mathrm{w}, 2-3$ times higher than that for the original sludge. Among the three configurations, configuration 3 enhanced sludge dewaterability the most.

In contrast, $\mathrm{N}_{2}$-freezing only slightly affected sludge dewaterability. This observation is consistent with our previous work (Jean et al., 1999; Chu et al., 1999).

Table 2

Experimental results: sewage sludge

\begin{tabular}{lccccc}
\hline & ZSV $(\mu \mathrm{m} / \mathrm{s})$ & CST $(\mathrm{s})$ & $h_{\mathrm{f}} / h_{\mathrm{I}}$ & Solid content $(\% \mathrm{w} / \mathrm{w})$ & Particle size $(\mu \mathrm{m})$ \\
\hline Original & 14 & 237 & 0.15 & 15.2 & 31.2 \\
Air-cooled & 150 & 44.9 & 0.14 & 20.5 & 153 \\
Configuration 1 & 90 & 38.7 & 0.11 & 19.4 & 115 \\
Configuration 2 & 26 & 46.0 & 0.17 & 17.3 & 88.8 \\
Configuration 3 & 37 & 38.3 & 0.18 & 27.9 & 102 \\
$\mathrm{~N}_{2}$ & 25 & 214 & 0.16 & 15.4 & 54.5 \\
\hline
\end{tabular}


Table 3

Experimental results: ferric hydroxide sludge

\begin{tabular}{lcclll}
\hline & ZSV $(\mu \mathrm{m} / \mathrm{s})$ & CST $(\mathrm{s})$ & $h_{\mathrm{f}} / h_{\mathrm{I}}$ & Solid content $(\% \mathrm{w} / \mathrm{w})$ & Particle size $(\mu \mathrm{m})$ \\
\hline Original & 94 & 51.8 & 0.35 & 14.5 & 61.7 \\
Air-cooled & 455 & 39.2 & 0.11 & 21.3 & 14.0 \\
Configuration 1 & 390 & 38.7 & 0.096 & 18.6 & 51.8 \\
Configuration 2 & 400 & 46.0 & 0.12 & 20.1 & 58.5 \\
Configuration 3 & 407 & 38.3 & 0.13 & 20.1 & 40.4 \\
$\mathrm{~N}_{2}$ & 350 & 45.3 & 0.14 & 19.8 & 63.8 \\
\hline
\end{tabular}

\subsection{Sewage sludge}

Consider the sewage sludge tests. The original sludge exhibited a CST of $237 \mathrm{~s}$ and ZSV of $14 \mu \mathrm{m} / \mathrm{s}$, indicating rather poor filterability and settleability. However, after $24 \mathrm{~h}$ of settling, its final sediment height reached a low level of $15 \%$. The solid content after filtration was $15.2 \% \mathrm{w} / \mathrm{w}$.

Air-cooled freezing of sewage sludge not only significantly increased the ZSV value $(150 \mu \mathrm{m} / \mathrm{s})$ and solid content $(21 \% \mathrm{w} / \mathrm{w})$, but also markedly lowered the corresponding CST (45 s). However, the final sediment height remained nearly unchanged. In addition, particle size increased from 31.2 to $153 \mu \mathrm{m}$ for the air-cooled sludge.

In contrast to the original sewage sludge, the dry ice-freezing also yielded a better dewaterability (although not as good as that for the air-cooled sludge). The CST for the treated sludge ranged from 38 to $46 \mathrm{~s}$. The ZSV values ranged from 26 to $90 \mu \mathrm{m} / \mathrm{s}$. Of particular interest is the high solid content that was reached by configuration 3 , almost 2.5 times to that of the original sludge. According to our results, the particle sizes increased to $89-115 \mu \mathrm{m}$, i.e. 3-4-times greater than the original sludge floc $(31.2 \mu \mathrm{m})$.

As illustrated in the activated sludge tests, $\mathrm{N}_{2}$-freezing only slightly affected the sludge characteristics. All dewatering indices were close to those of the original sludge. Furthermore, the particle size of the $\mathrm{N}_{2}$-frozen sludge was similar to that of the original sludge.

\subsection{Ferric hydroxide sludge}

The original ferric hydroxide sludge exhibited a me-

Table 4

Experimental results: oily sludge

\begin{tabular}{lll}
\hline & CST $(\mathrm{s})$ & Particle size $(\mu \mathrm{m})$ \\
\hline Original & 87.2 & 15.5 \\
Configuration 1 & 58.7 & 26.2 \\
Configuration 4 & 38.5 & 35.3 \\
\hline
\end{tabular}

dian ZSV of $94 \mu \mathrm{m} / \mathrm{s}$ and a CST of $52 \mathrm{~s}$. The final sediment height was $35 \%$ of the initial height. The solid content after filtration was $15 \% \mathrm{w} / \mathrm{w}$. In addition, the corresponding particle size was $61.7 \mu \mathrm{m}$.

Air-cooled freezing markedly enhanced the sludge's settleability $(\mathrm{ZSV}=455 \mu \mathrm{m} / \mathrm{s})$. The final sediment height reached only $11 \%$ of its initial height. In addition, although the solid content reached $21 \% \mathrm{w} / \mathrm{w}$, the particle size decreased rather than increased after freezing.

According to Table 3, all dewatering indices were close to those of the air-cooled sludge, indicating that configurations $1-3$ for the dry ice-freezing significantly improved the sludge dewaterability. Furthermore, $\mathrm{N}_{2^{-}}$ freezing also similarly improved the sludge dewaterability. For ferric hydroxide sludge freezing, the configuration used had only a slight effect on the sludge characteristics.

\subsection{Oily sludge}

Still settling of the original oily sludge for $24 \mathrm{~h}$ led to a clear supernatant and a deep-dark sediment. No oil phase was visually observed. The original oily sludge exhibited a median CST of $88 \mathrm{~s}$. Its particle size was not large $(15 \mu \mathrm{m})$.

Freezing can reduce the CST value and increase the particle size, indicating an enhancement of sludge deliquorability. Jean et al. (1999) noted, for the first time, that the freeze/thaw treatment of an oily sludge could separate the oil phase form the sludge body. A similar observation was also noted for the present sludge.

Table 4 lists the CST and particle size data for configurations 1 and 4. Notably, configuration 4 yielded a lower CST than that of configuration 1 . The corresponding particle size for the former was also larger than that for the latter. This may be attributed to the indirect thermal contact adopted in configuration 4 , which yielded a low freezing speed. However, the use of configuration 1 resulted in markedly improved deliquorability after freezing. 


\section{Freezing apparatus}

Experimental results for the four sludges of different characteristics indicated that: (1) all freezing tests markedly improved the sludge dewaterability; (2) although the air-cooled sludge generally exhibited the best dewaterability among all tests, the dry ice-freezing could condition the sludges to a sufficient level; and (3) among the dry ice-freezing tests the configuration 3 required the shortest freezing time (15-20 $\mathrm{min}$ ); however, it still yielded a satisfactory dewaterability. Such a configuration hence allows a high throughput (as discussed later). The squeezing action of ice layers developed on both sides of the sludge layer may explain this observation. (4) As is well known, ultra-fast freezing is not beneficial for oil separation or for filterability enhancement.

Freezing is generally an energy-intensive and a relatively slow process. Two issues are of relevant concern in industrial applications: cost analysis; and the freezing time required. In terms of cost, since CPC could obtain cold energy almost free of charge, and considering that freeze/thaw treatment could significantly enhance the dewatering rate of sludge, removal of the subsequent dewatering devices is possible. The fixed cost and the operating cost for sludge disposal could be markedly reduced. This freezing process is thus economically feasible.

Consider the freezing time, particularly for configuration 3 in the following sample calculation. With the present device, the freezing rate is estimated as $8-11$ $\mu \mathrm{m} / \mathrm{s}(1 \mathrm{~cm} / 15-20 \mathrm{~min})$. By taking $16 \mathrm{~h}$ daily of freezing as the basis of estimation, the achievable ice thickness is $0.48-0.64 \mathrm{~m} /$ day. A typical wastewater treatment plant produces 50 tons of sludge daily. The freezing bed area required is approximately $78-104 \mathrm{~m}^{2}$, or a 10-layer thermal contactor with each layer of size $3 \times 3 \mathrm{~m}$. Obviously, the current estimate is rather rough, and more detailed calculations could be made according to a heat transfer model such as that proposed by Vesilind and Martel (1990). We cannot necessarily conclude that the bench-scale test adopted in this work could be satisfactorily scaled up to full scale. Nevertheless, the simple analysis adopted herein can still be viewed as an order-of-magnitude estimate for the required size of the proposed contactor. We believe such a contactor would be compact and easy to construct and operate.

\section{Conclusions}

Dry ice was used as the heat sink in sludge freeze/thaw treatment. Four distinct sludges, i.e. a waste activated sludge, a sewage sludge, $\mathrm{Fe}(\mathrm{OH})_{3}$ sludge, and an oily sludge, were frozen at four-thermal contact configurations with dry ice. Experimental results indicate that the dry ice-freezing could sufficiently condition these sludges. All the configurations for dry ice-freezing tests yielded significant enhancement of dewaterability. The zone settling velocity (ZSV) ranged from 76 to $150 \mu \mathrm{m} / \mathrm{s}$, capillary suction time (CST) was less than $36 \mathrm{~s}$, sediment ratios $\left(h_{\mathrm{f}} / h_{\mathrm{i}}\right)$ were reduced to $0.25-0.55$ and the solid contents all exceeded $13.6 \%$ $\mathrm{w} / \mathrm{w}$, which are 2-3-times higher than that for the original sludge. Among the three configurations, configuration 3 enhanced sludge dewaterability the most. In contrast, $\mathrm{N}_{2}$-freezing only slightly affected sludge dewaterability. Since the Chinese Petroleum Corporation has free cold-energy for freezing the sludge, the use of dry ice is economically feasible. A 10-layer thermal contactor with each layer of size $3 \times 3 \mathrm{~m}$ could achieve thermal contact.

\section{Acknowledgements}

The Chinese Petroleum Corporation (CPC) and the National Science Council, ROC supported this project.

\section{References}

Chen, G.W, Chang, I.L, Hung, W.T., Lee, D.J., 1996a. Regimes of zone settling of waste activated sludges. Wat. Res. 30, 1844-1850.

Chen, G.W., Chang, I.L., Hung, W.T., Lee, D.J., 1996b. Effects of freeze/thaw treatment on zone settling of waste activated sludges. J. Envir. Sci. Hlth. A 31, 521-531.

Chu, C.P., Feng, W.H., Tsai, Y.H., Lee, D.J, 1997. Unidirectional freezing of waste activated sludge: the presence of sodium chloride. Envir. Sci. Tech. 31, 1512-1517.

Chu, C.P., Feng, W.H., Chang, B.V., Lee, D.J., 1999. Reduction in microbial density level through freezing and thawing. Wat. Res. 33, 3532-3535.

Hanisch, C., 1999. Exploring options for CO2 capture and management. Envir. Sci. Tech. 33, 66A-70A.

Hung, W.T, Chang, I.L, Lin, W.W, Lee, D.J., 1996. Unidirectional freezing of waste activated sludge: effects of freezing speed. Envir. Sci. Tech. 30, 2391-2396.

Hung, W.T., Feng, W.H., Tsai, I.H., Lee, D.J., Hong, S.G., 1997. Unidirectional freezing of waste activated sludge: radial freezing vs. vertical freezing. Wat. Res. 31, 2219-2228.

Jean, D.S, Lee, D.J., 1999. Effects of electrolyte and curing on freeze/thaw treatment of ferric hydroxide sludge. Colloids Surf. A 162, 285-288.

Jean, D.S, Lee, D.J, Wu, J.C.S, 1999. Separating oil from oily sludge by freezing and thawing. Wat. Res. 33, 1756-1759. 
Lee, D.J., Hsu, Y.H., 1992. Fluid flow in capillary suction apparatus. Ind. Eng. Chem. Res. 31, 2379-2384.

Lee, D.J., Hsu, Y.H., 1993. Cake formation in capillary suction apparatus. Ind. Eng. Chem. Res. 32, 1180-1185.

Lee, D.J., Hsu, Y.H., 1994. Fast freeze/thaw treatment on activated sludge: floc Structure and sludge dewaterability. Envir. Sci. Tech. 28, 1444-1449.

Martel, C.J., Affleck, R., Yushak, M., 1998. Operational parameters for mechanical freezing of alum sludge. Wat. Res. 32, 2464-2654.
Parker, P.J., Collins, A.G., 1999. Ultra-rapid freezing of water treatment residuals. Wat. Res. 33, 2239-2246.

Vesilind, P.A., Martel, C.J., 1990. Freezing of water and wastewater sludges. J. Envir. Eng. ASCE 116, 854-862.

Vesilind, P.A., Wallinmaa, S., Martel, C.J., 1991. Freeze-thaw sludge conditioning and double layer compression. Can. J. Civil Eng. 18, 1078-1083. 